\title{
Preparation of Gold Nanoparticles for Biomedical Applications Using Chemometric Technique
}

\author{
Soheila Honary ${ }^{1 *}$, Pouneh Ebrahimi ${ }^{2}$ and Maedeh Ghasemitabar ${ }^{1}$ \\ ${ }_{1}^{1}$ Mazandaran University of Medical Sciences, School of Pharmacy, Pharmaceutical Sciences Research Center, \\ Sari, ${ }^{2}$ Gonbad-Kavous University, PO Box 163, Gonbad, Iran
}

*For correspondence: Email: Shonary@yahoo.com; honary@ualberta.ca

\begin{abstract}
Purpose: To study the effect of process conditions on the size of gold nanoparticles (AuNPs) prepared by chemometric technique.

Methods: AuNPs were prepared by adding $5 \mathrm{ml}$ of $5 \mathrm{mM}$ of gold (III) chloride hydrate

$\mathrm{HAuCl}_{4}(2 \mathrm{mg} / \mathrm{mL})$ to $85 \mathrm{ml}$ of filtered deionized water, then refluxed in a $250 \mathrm{~mL}$ flask over a hot plate and heated to boiling point. Five milliliters of sodium citrate solution of varying concentrations were quickly added to the boiling solution and stirred for 30 min until the color turned to wine red. Chemometric approach, based on multivariant analysis, was applied to the optimization of iron oxide nanoparticle size in respect of three parameters, viz, concentration of sodium citrate solution, stirrer speed and ionic strength of the medium. The experiments were performed according to Box-Behnken experimental design.

Results: The regression model obtained was characterized by both descriptive and predictive ability. The method was optimized with respect to average diameter as a response. The average diameter of the nanoparticles produced under different conditions were between 17.7 up to $168.8 \mathrm{~nm}$. The criteria for the evaluation of the descriptive capability of a polynomial were $R^{2}=0.974$, standard error $=13.994$ and F-ratio $=18.4$.

Conclusion: It can be concluded that the Box-Behnken experimental design provides a suitable approach for optimizing and testing the robustness of gold nanoparticle preparation method.
\end{abstract}

Keywords: Box-Behnken design, Optimization, Nanoparticles, Gold, Biomedical, Chemometric.

Tropical Journal of Pharmaceutical Research is indexed by Science Citation Index (SciSearch), Scopus, International Pharmaceutical Abstract, Chemical Abstracts, Embase, Index Copernicus, EBSCO, African Index Medicus, JournalSeek, Journal Citation Reports/Science Edition, Directory of Open Access Journals (DOAJ), African Journal Online, Bioline International, Open-J-Gate and Pharmacy Abstracts

\section{INTRODUCTION}

Ultra-fine metal and metal alloy powders have many impotent applications in electronics, dental, and chemical industries [1-4]. Gold nanoparticles are promising nanocarriers for therapeutics due to their unique optical, chemical, and biological properties [5,6]. Gold nanospheres can be synthesized by controlled reduction of an aqueous $\mathrm{HAuCl}$ solution using different reducing agents under varying conditions. The most commonly used reducing agent is citrate, which can produce nearly mono-disperse gold nanospheres $[7,8]$.

Optimizing refers to improving the performance of a system, a process, or a product in order to obtain the maximum benefit from it $[9,10]$. Experimental design and optimization are tools that are used to systemically examine different types of problems that arise in research, development and production. It is obvious that if experiments are performed randomly, the results 
obtained will also be random. Therefore, after defining experimental variables and responses, experiments can be planned and performed in such a way that maximum information is gained from a minimum of experiments [11].

In recent years, chemometric tools have been frequently applied to the optimization of analytical methods, considering their advantages such as a reduction in the number of experiments that need be executed resulting in lower reagent consumption and considerably less laboratory work. Furthermore, these methods allow the development of mathematical models that permit assessment of the relevance as well as statistical significance of the factor effects being studied as well as evaluate the interaction effects between the factors [12].

Response surface methodology (RSM) is the most relevant multivariate techniques used in optimization. This methodology is a collection of mathematical and statistical techniques based on the fit of a polynomial equation to the experimental data, which must describe the behavior of a data set with the objective of making statistical previsions [10]. It can well be applied when a response or a set of responses of interest are influenced by several variables. The objective is to simultaneously optimize the levels of these variables to attain the best system performance. In the present work, we have employed RSM for optimization of gold nanoparticle (AuNP) preparation. Predict the best condition for production of smaller gold nanoparticles is our purpose as well.

\section{EXPERIMENTAL}

\section{Chemicals}

Gold (III) chloride hydrate $\left(\mathrm{HAuCl}_{4}\right)$ and other chemical reagents used were purchased from Merck (Germany).

\section{Preparation of gold nanoparticles (AuNPs)}

AuNPs were prepared as described elsewhere [13]. Briefly, $85 \mathrm{ml}$ of filtered deionized water was refluxed in $250 \mathrm{~mL}$ flask on a hot plate. Five milliliters of $5 \mathrm{mM} \mathrm{HAuCla}(2 \mathrm{mg} / \mathrm{mL})$ was added to the above refluxed water and heated to boiling point. Five milliliters of $25 \mathrm{mM}$ sodium citrate solution was quickly added to the boiling solution and stirred for $30 \mathrm{~min}$ until the color turned to wine red.
Determination of nanoparticle size and polydispersity

Size and polydispersity of particles were determined with a zetasizer nano particle analyzer using (model 3600, Malvern Instruments, UK)) at $25^{\circ} \mathrm{C}$ and scattering angle of $90^{\circ}$.

\section{Experimental design}

Before applying the RSM methodology, it was first necessary to choose an experimental design that will define which experiments should be carried out in the experimental region being studied. There are some experimental matrices for this purpose. Experimental designs for firstorder models (e.g., factorial designs) can be used when the data set does not present curvature. However, to approximate a response function to experimental data that cannot be described by linear functions, experimental designs for quadratic response surfaces should be used, such as three level factorial, namely, Doehlert design and Box-Behenken central composite. In this study, the latter design was used.

Nanoparticles were prepared with different combinations of the values of the three variables according to the Box-Behenken experimental design. The three experimental variables considered were: stirring rate (S, RPM), sodium citrate concentration $(\mathrm{C}, \mathrm{mM})$ and ionic strength (IS, mM). All experiments were carried out in duplicate and the sequence of experiments was randomized. Three experimental factors were studied at three levels (Table 1). These factors were chosen as they were considered to have the most significant effect on the size of nanoparticles. The levels were selected based on knowledge acquired from initial experimental trials. In this design, the experiment in the central point provided a more precise estimate of pure experimental error and is a measure of the adequacy of the model (lack of fit). All statistical analyses were performed on range-scaled factor values of $-1,+1$ with SPSS 16 software.

Table 1: Three studied factors and the corresponding three level settings

\begin{tabular}{cccc}
\hline Level & $\mathbf{S}^{\mathrm{a}}$ & $\mathbf{C}^{\mathrm{b}}$ & $\mathbf{I S}^{\mathrm{c}}$ \\
\hline-1 & 750 & 1 & 0 \\
0 & 1000 & 25 & 25 \\
+1 & 1250 & 49 & 50 \\
\hline
\end{tabular}

${ }^{a} S, C$ and IS refer to stirring rate $(\mathrm{rpm})$, sodium citrate concentration $(\mathrm{mM})$ and ionic strength (mmole), respectively. 


\section{RESULTS}

The effect of three factors on average diameter of gold nanoparticles was studied. The experiments were performed in a random order on three levels for each factor using a BoxBehenken design whicht provides enough information for calculation of the regression model containing linear, interactions and curved factor effects. The values of response data for the experiments were also presented in Table 2.

Table 2: Experimental conditions for Box-Behenken design and mean response for particle size

\begin{tabular}{ccccc}
\hline Trial & S & C & IS & $\begin{array}{c}\text { Mean } \\
\text { diameter } \\
\text { (nm) }\end{array}$ \\
\hline 1 & + & + & 0 & 65.085 \\
2 & + & - & 0 & 128.3 \\
3 & - & + & 0 & 116.555 \\
4 & - & - & 0 & 26.645 \\
5 & + & 0 & + & 168.85 \\
6 & + & 0 & - & 56.18 \\
7 & - & 0 & + & 113.2 \\
8 & - & 0 & - & 116.355 \\
9 & 0 & + & + & 151.85 \\
10 & 0 & + & - & 17.735 \\
11 & 0 & - & + & 77.99 \\
12 & 0 & - & - & 102.9 \\
13 & 0 & 0 & 0 & 143.1 \\
14 & 0 & 0 & 0 & 100.305 \\
15 & 0 & 0 & 0 & 104.056 \\
\hline
\end{tabular}

The mean diameter of nanoparticles for all 15 experiments was fitted to the polynomial model. The coefficients of the model were calculated by backward multiple regression technique and validated by the analysis of variance (ANOVA). The criteria for the evaluation of descriptive capability of a polynomial were Fisher-ratio value $(F)$, adjusted $R^{2}$ and standard error of estimation (SE). It was found that the model which successfully described the size of nanoparticles were polynomials containing various number of terms and different combinations of factors. The related model and its statistics are presented in Table 3.

\section{DISCUSSION}

The results of ANOVA demonstrated that the largest effect on size of nanoparticles were due to interaction between citrate concentration (C), ionic strength (IS) and stirring rate (S). No significant effect was observed due to the individual effects of these factors. However, two interactions of these factors show significant effect on the mean size of nanoparticles. It is interesting to demonstrate that in this study, the quadratic term of citrate concentration was also very significant in influencing the size of the nanoparticles. This means that the respective response hyper-surfaces in the multidimensional factor space are curved in the sphere of the experimental design.

Table 3: Intercept, coefficients and mean effects for the predictive model obtained for three factors

\begin{tabular}{ccc}
\hline Variable & Coefficient & Mean effect \\
\hline Intercept & 114.578 & - \\
$C^{2}$ & -28.696 & -0.340 \\
$\mathrm{~S} \times \mathrm{C}$ & -38.281 & -0.470 \\
$\mathrm{~S} \times \mathrm{IS}$ & 28.956 & 0.355 \\
$\mathrm{C} \times \mathrm{IS}$ & 39.756 & 0.488 \\
$\mathrm{~S}^{2} \times I S$ & 27.379 & 0.336 \\
$\mathrm{C}^{2} \times \mathrm{S}$ & 12.546 & 0.154 \\
$\mathrm{C}^{2} \times I S$ & 27.301 & 0.335 \\
\hline$R^{2}=0.974$, standard error $=13.994$, and F-ratio $=18.4$
\end{tabular}

The existence of second-order interactions between the principal factors in conditions of our experiments emphasizes the necessity to carry out active multi-factor experiments for optimization of the preparation process of nanoparticles. This indicates that the use of simultaneous method is essential for optimization of gold nanoparticle preparation process. The size of nanoparticles in other experimental conditions was predicted using the equation obtained. When the optimum conditions were tested and the experimental results compared to predicted results, there was a good agreement between the predicted and experimental results for 13 experimental conditions not entered in the modeling (Fig 1). To assess the suitability of obtained MLR model, the residuals were analyzed statistically, and as Fig 2 shows, the fitting of this model and the model prediction ability is satisfactory.

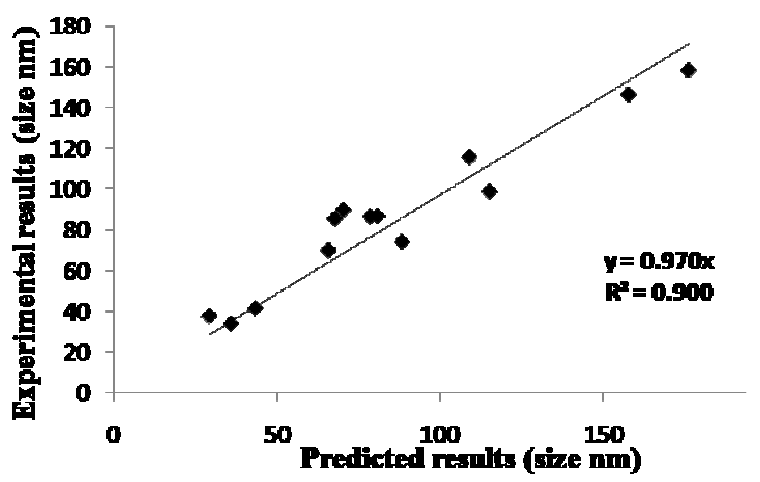

Fig 1: Predicted vs. experimental gold nanoparticle size for 13 experimental conditions not included in the modeling. 


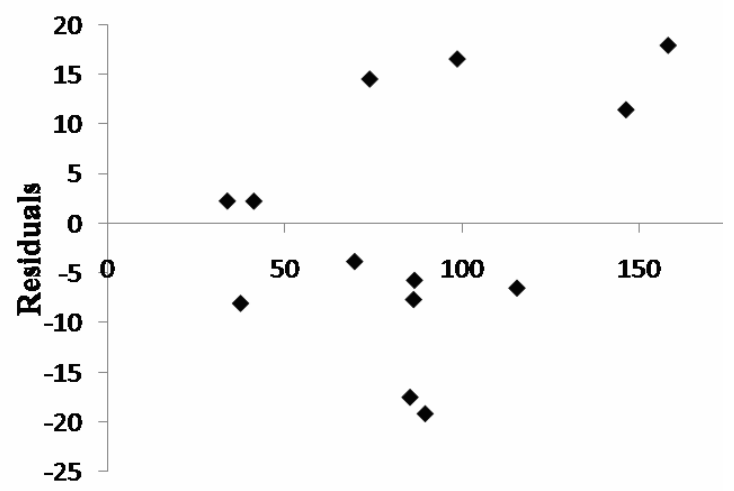

Experimental results (size.nm)

Fig 2: The residual plot for the predicted size of gold nanoparticles according to the regression model reported in Table 3.

\section{CONCLUSION}

A chemometric approach allows the researcher to determine the best conditions for the preparation of gold nanodispersions using a limited number of experiments. The results showed that particle size regularly changes as a function of citrate concentration, ionic strength and stirring rate. Furthermore, the Box-Behnken design permits the determination of a regression model which describes the dependence of particle size on the experimental parameters employed in the preparation of gold nanoparticles. The existence of second-order interactions between the principal factors in the conditions of our experiments emphasizes the necessity of using simultaneous method for the optimization of gold nanoparticle preparation process.

\section{ACKNOWLEDGEMENT}

This work was financially supported by Mazandaran University of Medical Sciences, Sari, Iran.

\section{REFERENCES}

1. Wang S, Lu W, Tovmachenko O, Rai US, Yu H, Ray $P C$, Challenge in understanding size and shape dependent toxicity of gold nanomaterials in human skin keratinocytes, Chem. Phys. Lett. 2008; 463: 145-149.

2. Bhattacharya $R$, Mukherjee $P$, Biological properties of "naked" metal nanoparticles, Adv. Drug Deliver. Rev. 2008; 60; 1289-1306.

3. Kima KD, Hanb DN, Kimb HT, Optimization of experimental conditions based on the Taguchi robust design for the formation of nano-sized silver particles by chemical reduction method, Chem. Eng. J. 2004; 104: 55-61.

4. Du Y, Luo X, Xu JJ, Chen $H Y$, A simple method to fabricate a chitosan-gold nanoparticle film and its application in glucose biosensor, Bioelectrochemistry 2007; 70: 342-347.

5. Kim CK, Ghosh P, Rotello VM, Multimodal drug delivery using gold nanoparticles, Nanoscale 2009; 1: 6167.

6. Pissuwan D, Niidome $T$, Cortie MB, The forthcoming applications of gold nanoparticles in drug and gene delivery systems, J. Control. Release 2011; 149: 65-71.

7. Cai W, Gao T, Hong H, Sun J, Applications of gold nanoparticles in cancer nanotechnology, Nanotechnology, 2008; 1: 17-32.

8. Sonavane G, Tomada $K$, Sano A, Ohshima $H$, Terada $H$, Makino $K$, Invitro permeation of gold nanoparticles through rat akin and rat intestine: effect of particle size, colloid surface $B$ 2008; 65 : 1 10.

9. Prashant K. Jain, Kyeong Seok Lee, Ivan H. El-Sayed, Mostafa A. El-Sayed, calculated absorption and scattering properties of gold nanoparticles of different size, shape, and composition: applications in biological imaging and biomedicine, J. Phys. Chem. B 2006; 110: 7238-7248.

10. Bezerraa MA, Santelli RE, Oliveiraa EP, Villar LS, Escaleiraa LA, Response surface methodology (RSM) as a tool for optimization in analytical chemistry, Talanta 2008; 76: 965-977.

11. Lundstedt $T$, Seifert E, Boman A, Lek PM, Experimental design and optimization, chemometr. Intell. Lab. 1998; 42: 3-40.

12. Ferreira SL, Bruns RE, Ferreira HS, Matos GD, David JM, Brandão GC, da Silva EG, Portugal LA, dos Reis PS, Souza AS, dos Santos WN, Box-Behnken design: An alternative for the optimization of analytical methods, Anal. Chim. Acta. 2007; 597: 179-186.

13. Sonavane G, Tonoda K, Makino K, Biodistribution of colloidal gold nanoparticles after intravenous administration: Effect of particle size, colloid surface $B$ 2008; 66: 274-280. 